PROCEEDINGS OF THE

AMERICAN MATHEMATICAL SOCIETY

Volume 128, Number 2, Pages 445-451

S 0002-9939(99)04989-8

Article electronically published on October 12, 1999

\title{
COMPLEMENTED IDEALS IN THE FOURIER ALGEBRA OF A LOCALLY COMPACT GROUP
}

\author{
PETER J. WOOD
}

(Communicated by Dale Alspach)

\begin{abstract}
In this paper we provide a necessary condition for a closed ideal in the Fourier algebra of a locally compact amenable group to be completely complemented. The classification of completely complemented ideals is completed in the case of an amenable discrete group. We also investigate the ideals possessing a bounded approximate identity.
\end{abstract}

\section{INTRODUCTION}

In [19] W. Rudin and in [18] H. Rosenthal investigated the problem of classifying which closed ideals in the group algebra $L^{1}(G)$ of a locally compact abelian group are complemented. Using the amenability properties of the abelian group $G$, Rosenthal was able to show that if a closed ideal in $L^{1}(G)$ is complemented, then its hull (or zero set) is contained in the closed coset ring of $\hat{G}$. His method involved the construction of an invariant projection onto the annihilator of the ideal. In [16] T.-S. Liu, A. van Rooij and J.-K. Wang continued the investigation, and considered the connection between ideals possessing bounded approximate identities and invariant complementation.

We recall that a Banach algebra $\mathcal{A}$ is Banach algebra amenable (or simply amenable if no confusion arises) if each bounded derivation $D$ from $\mathcal{A}$ to a dual Banach $\mathcal{A}$-bimodule $X^{*}$ is inner. In [11] B. Johnson showed that the group algebra of a locally compact group $L^{1}(G)$ is amenable if and only if the group $G$ is amenable. It is possible to recover the major results of [18] and [16] by connecting Johnson's result with general properties of amenable Banach algebras (see [14] and [6]). In this paper, we would like to find a similar connection in the Fourier algebra. Unfortunately however, the Fourier algebra $A(G)$ is not Banach algebra amenable for all amenable groups $G$ (see [12]).

In [20], Ruan showed that the "appropriate" definition of amenability for $A(G)$ is that of operator amenability. In this paper, our approach will be to exploit the analogous Banach algebra amenability properties for the category of operator spaces, to achieve results similar to those of Rosenthal's.

Received by the editors August 18, 1996 and, in revised form, March 10, 1998.

2000 Mathematics Subject Classification. Primary 46J20, 46L07; Secondary 43A30, 46H25.

Key words and phrases. Fourier algebra, operator spaces, operator amenability, complemented ideals, bounded approximate identities.

(C)1999 American Mathematical Society 


\section{Preliminaries}

By an operator space $X$ we mean a norm closed subspace of $B(H)$ for some Hilbert space $H$. (See [8] for an abstract characterization of operator spaces.) Given a bounded linear map $T: X \mapsto Y$ between operator spaces $X$ and $Y$ we get the induced maps $T^{(n)}: M_{n}(X) \mapsto M_{n}(Y)$ given by $T^{(n)}\left(\left[x_{i j}\right]\right)=\left[T\left(x_{i j}\right)\right]$. $T$ is called completely bounded if sup $\left\|T^{(n)}\right\|$ is finite, and we define $\|T\|_{c b}=\sup \left\|T^{(n)}\right\|$. We shall say $T$ is completely contractive if $\|T\|_{c b} \leq 1$. Note that if $T$ is completely bounded, so is its adjoint $T^{*}$. Furthermore $\|T\|_{c b}=\left\|T^{*}\right\|_{c b}$. A Banach algebra $\mathcal{A}$ which is also an operator space is called a completely contractive Banach algebra if the natural multiplication $m: \mathcal{A} \hat{\otimes} \mathcal{A} \mapsto \mathcal{A}$ is completely contractive with respect to the operator space projective tensor product $\hat{\otimes}[20]$.

Let $\mathcal{A}$ be a Banach algebra. A Banach $\mathcal{A}$-bimodule is a two sided $\mathcal{A}$-bimodule $X$ which is itself a Banach space and which also satisfies

$$
\|a x\|_{X} \leq\|a\|_{\mathcal{A}}\|x\|_{X} \quad \text { and } \quad\|x a\|_{X} \leq\|a\|_{\mathcal{A}}\|x\|_{X}
$$

for each $a \in \mathcal{A}$ and each $x \in X$. If in addition, $\mathcal{A}$ is a completely contractive Banach algebra and $X$ is an operator space such that the $\mathcal{A}$-bimodule operations $(a, x) \mapsto a x$ and $(a, x) \mapsto x a$ are complete bounded from $\mathcal{A} \hat{\otimes} X$ onto $X$, then $X$ is called an operator $\mathcal{A}$ - bimodule.

The sequence

$$
\Sigma: 0 \mapsto X \stackrel{f}{\mapsto} Y \stackrel{g}{\mapsto} Z \mapsto 0
$$

is called exact if $f$ is one-to-one, $g$ is onto and $i m f=k e r g$ for bounded linear maps $f$ and $g$. We wish to consider such sequences in both the category of Banach spaces and the category of operator spaces. An exact sequence of Banach spaces is called admissible if there exist bounded maps $F: Y \mapsto X$ and $G: Z \mapsto Y$ such that $F f=i d_{X}$ and $g G=i d_{Z}$. (We call $F$ a left inverse for $f$ and $G$ a right inverse for $g$.) If $X, Y$ and $Z$ are Banach $\mathcal{A}$-bimodules, then we say the sequence $\Sigma$ splits if there exist a bounded left inverse for $f$ and a bounded right inverse for $g$ which are module maps. In [6] it was shown that the existence of a right inverse for $g$ is equivalent to the existence of a left inverse for $f$. (Note this is false in the category of operator spaces - see remarks after Theorem 1.) If $X, Y$ and $Z$ are operator spaces, we assume the maps $f$ and $g$ are completely bounded, and we say $\Sigma$ is completely admissible if there exist completely bounded left and right inverses for $f$ and $g$ respectively. If $X, Y$ and $Z$ are operator $\mathcal{A}$-bimodules, we say it completely splits if there exist completely bounded left and right inverses for $f$ and $g$ respectively which are also module maps.

For a given locally compact group $G$, the Fourier algebra denoted $A(G)$ was introduced in [9] by P. Eymard, and consists of the coefficient functions of the left regular representation $\lambda$ of $G$, i.e.

$$
A(G)=\left\{u(x)=\langle\lambda(x) \zeta, \eta\rangle: \zeta, \eta \in L^{2}(G)\right\} .
$$

It is well known that $A(G) \cong L^{1}(\hat{G})$ when $G$ is abelian and $\hat{G}$ is its dual group. The dual space of $A(G)$ is the left group von Neumann algebra $V N(G)$, and $A(G)$ is a commutative Banach algebra with pointwise multiplication (see [9]). Thus regarded as the predual of an operator space, $A(G)$ has a natural operator space structure under which $A(G)$ is a completely contractive Banach algebra (see [20]). 
Given an operator space $X$ it is well known that every bounded linear functional is completely bounded. Thus we can identify $X^{*}$ with $C B(X, C)$ the space of completely bounded linear maps from $X$ into $C$. In addition, we may view $X^{*}$ as an operator space via the identification $M_{n}(C B(X, C))=C B\left(X, M_{n}(C)\right)$ (see [5]).

In [20], Ruan introduced the notion of operator amenability for completely contractive Banach algebras. A completely contractive Banach algebra $\mathcal{A}$ is called operator amenable if every completely bounded derivation $D$ of $\mathcal{A}$ into a dual operator $\mathcal{A}$-bimodule $X^{*}$ is inner. In [20] it is shown that $A(G)$ is operator amenable if and only if $G$ is amenable, establishing the natural analogue of Johnson's result for group algebras.

It is easy to verify that given operator $\mathcal{A}$-bimodules $X$ and $Y$, the space $C B(X, Y)$ of completely bounded maps from $X$ to $Y$ can be regarded as an operator $\mathcal{A}$ bimodule via the following module operations

$$
(a \cdot T)(x)=a \cdot(T(x)) \text { and }(T \cdot a)(x)=T(a \cdot x)
$$

where $T \in C B(X, Y), a \in \mathcal{A}$, and $x \in X$.

A subspace $Y$ of a Banach space $X$ is said to be complemented if there exists a bounded projection $P$ from $X$ onto $Y$. If $X$ is an operator space, we say $Y$ is completely complemented if $P$ can be chosen to be completely bounded.

\section{Splitting of exact Sequences}

The following theorem is the operator space analogue of [6], Theorem 2.3. The proof is similar, but we include it for completeness.

Theorem 1. Let $\mathcal{A}$ be an operator amenable Banach algebra, and let

$$
\Sigma: 0 \mapsto X^{*} \stackrel{f}{\mapsto} Y \stackrel{g}{\mapsto} Z \mapsto 0
$$

be a completely admissible short exact sequence of operator $\mathcal{A}$-bimodules with $X^{*}$ a dual operator $\mathcal{A}$-bimodule. Then $\Sigma$ splits completely.

Proof. Since $\Sigma$ is completely admissible, there exists $G \in C B(Z, Y)$ such that $g G=i d_{Z}$. Define

$$
D(a)=a \cdot G-G \cdot a
$$

Clearly $D$ is a completely bounded derivation of $A$ to the bimodule $C B(Z, Y)$. Now since $g$ is a module map,

$$
g(D a(z))=g(a \cdot G-G \cdot a)(z)=a \cdot z-a \cdot z .
$$

Thus $D(\mathcal{A}) \subset C B(Z, \operatorname{kerg})=C B(Z, i m f)$. Since $\Sigma$ is completely admissible, the derivation given by

$$
f^{-1} D: \mathcal{A} \mapsto C B\left(Z, X^{*}\right) \cong(Z \hat{\otimes} X)^{*}
$$

is completely bounded (see [20] for the last identification). Since $\mathcal{A}$ is operator amenable, $f^{-1} D$ is inner. Thus there exists $Q \in C B\left(Z, X^{*}\right)$ such that

$$
f^{-1} D(a)=a \cdot Q-Q \cdot a .
$$

Since $f$ is a module map, we have

$$
D(a)=a \cdot f Q-f Q \cdot a=a \cdot G-G \cdot a .
$$


Let $T=G-f Q$. Clearly $T$ is a module map from $Z$ to $Y$ and since $\operatorname{Im} f=\operatorname{Kerg}$ we have

$$
g T(z)=g G(z)-g f Q(z)=g G(z)=z .
$$

Now define $F: Y \mapsto X^{*}$ by

$$
F(y)=f^{-1}(y-T g(y)) \quad \forall y \in Y .
$$

Clearly $F f=i d_{X^{*}}$, and $F$ is a completely bounded module map. Thus $\Sigma$ splits completely.

Remark. At first glance, one might hope that the important results concerning Banach algebra amenability translate directly to the operator space setting immediately, with little more than the insertion of the word "completely" where appropriate. However the situation is considerably more subtle than that. To illustrate this, note that unlike in the general Banach space case, the existence of a completely bounded right inverse for $g$ does not imply the existence of a completely bounded left inverse for $f$. Consider the sequence

$$
\Sigma: 0 \mapsto M A X(X) \stackrel{f}{\mapsto} M I N(X) \stackrel{g}{\mapsto} 0 \mapsto 0
$$

where $g$ is the zero map and $f$ the identity. Here $M A X(X)$ and $M I N(X)$ denote the maximal and minimal operator space structures on a general normed space $X$ (see [7]). Note the identity map from $M A X(X)$ to $M I N(X)$ is completely bounded (in fact every bounded map from any operator space into $M I N(X)$ is completely bounded; see [2]) while its inverse is not completely bounded in general (see [17]). Obviously the zero map is a completely bounded right inverse for $g$. In particular this means that the analogue of Proposition 1.1 of [6] fails in the category of operator spaces. The author would like to thank David Blecher for directing us to this example.

Suppose $\mathcal{A}$ is an operator amenable Banach algebra, and let $\mathcal{J}$ be a closed ideal. We wish to consider the sequence

$$
\Sigma: 0 \mapsto \mathcal{J} \stackrel{i}{\mapsto} \mathcal{A} \stackrel{v}{\mapsto} \mathcal{A} / J \mapsto 0
$$

and its dual sequence

$$
\Sigma^{*}: 0 \mapsto \mathcal{J}^{\perp} \stackrel{v^{*}}{\mapsto} \mathcal{A}^{*} \stackrel{i^{*}}{\mapsto} \mathcal{J}^{*} \mapsto 0,
$$

where $i$ is the inclusion map, $v$ is the canonical quotient map, and $i^{*}, v^{*}$ are their adjoints. Clearly $i$ and $v$ are completely bounded module maps and thus so are $i^{*}$ and $v^{*}$. (Note that $(\mathcal{A} / J)^{*} \cong J^{\perp}$.)

Now suppose that $\mathcal{J}$ is completely complemented by a projection $P: \mathcal{A} \mapsto J$. We define a map $Q: \mathcal{A} / J \mapsto \mathcal{A}$ by $Q(a+J)=a-P(a)$. Note that $Q$ is well defined since if $a+J=b+J$, then $a-b \in J$ so $Q(a-b+J)=a-b-P(a-b)=0$. Furthermore $Q$ is completely bounded and a left inverse for $v$. Thus we conclude the exact sequence $\Sigma^{*}$ is completely admissible. Recall that a submodule $Y$ of an $\mathcal{A}$-module $X$ is called invariantly complemented if there exists a projection $T$ onto $X$ which commutes with the module action, i.e. $T: X \mapsto Y$ such that $T(a x)=a T(x)$ for all $a \in \mathcal{A}$ and $x \in X$. Applying Theorem 1 we have

Theorem 2. Let $\mathcal{J}$ be a closed ideal in an operator amenable Banach algebra. If $\mathcal{J}$ is completely complemented, then $\mathcal{J}^{\perp}$ is invariantly completely complemented. 
Now we are ready to provide the connection between complete invariant complementation and bounded approximate identities in the operator space category.

Theorem 3. Let $\mathcal{A}$ be an operator amenable Banach algebra, and $\mathcal{J}$ a closed ideal. Then $\mathcal{J}^{\perp}$ is completely complemented if and only if $\mathcal{J}$ has a bounded approximate identity.

Proof. Assume $\mathcal{J}^{\perp}$ is complemented by a completely bounded projection. Then by Theorem 1, $\Sigma^{*}$ splits completely. In particular $\Sigma^{*}$ splits, and hence by standard Banach algebra arguments, $\mathcal{J}$ has a bounded approximate identity (see [6]).

For the converse we follow [6], Proposition 3.5. If $\mathcal{J}$ has a bounded approximate identity, let $\Phi$ be a weak-* limit point in $\mathcal{J}^{* *}$. Define $S: \mathcal{J}^{*} \mapsto \mathcal{A}^{*}$ by

$$
\langle S(\phi), a\rangle=\langle\Phi, \phi \cdot a\rangle \quad \forall \phi \in \mathcal{J}^{*} .
$$

Clearly $i^{*} S=i d_{\mathcal{J}^{*}}$. Now define an operator $T$ on $\mathcal{A}^{*}$ by

$$
T(\phi)=\phi-S i^{*}(\phi) .
$$

Routine calculations show that if $x \in \mathcal{J}$, we have

$$
\langle T \phi, x\rangle=\langle\phi, x\rangle-\left\langle S i^{*} \phi, x\right\rangle=0
$$

and if $\phi \in \mathcal{J}^{\perp}$, then $T \phi=\phi$. In particular we see that $T$ is a projection onto $\mathcal{J}^{\perp}$. It now suffices to show that $S$ is completely bounded (whence $T$ is). Now

$$
\begin{aligned}
& \left\|S^{(n)}\right\|=\sup _{\left\|\left[\phi_{i j}\right]\right\| \leq 1}\left\|\left[S\left(\phi_{i j}\right)\right]\right\| \quad\left[\phi_{i j}\right] \in M_{n}\left(\mathcal{J}^{*}\right) \\
& =\sup _{\left\|\left[\phi_{i j}\right]\right\| \leq 1} \sup _{\|a\| \leq 1}\left\|\left\langle S\left(\phi_{i j}\right), a\right\rangle\right\|=\sup _{\left\|\left[\phi_{i j}\right]\right\| \leq 1} \sup _{\|a\| \leq 1}\left\|\left\langle\Phi, \phi_{i j} \cdot a\right\rangle\right\| \\
& =\sup _{\left\|\left[\phi_{i j}\right]\right\| \leq 1} \sup _{\|a\| \leq 1}\left\|\Phi^{(n)}\left[\phi_{i j} \cdot a\right]\right\| \\
& \leq\|\Phi\| \text {. }
\end{aligned}
$$

To see the last inequality, note that $\Phi$ is a linear functional on $\mathcal{J}^{*}$ and hence is completely bounded with $\|\Phi\|_{c b}=\|\Phi\|$ and we also have that the module action is completely contractive. Hence $S$ is completely bounded and the result is proven.

Remark. Notice that the operator amenability of the algebra was not necessary in the construction of the map $T$. Since $T$ is a module map, we can conclude in general that if $\mathcal{J}$ possesses a bounded approximate identity, then $\mathcal{J}^{\perp}$ is completely invariantly complemented.

The next corollary now follows easily.

Corollary 4. If $\mathcal{J}$ is a completely complemented closed ideal in an operator amenable Banach algebra, then $\mathcal{J}$ has a bounded approximate identity.

\section{IdEALS IN the Fourier Algebra}

Given an element $u \in A(G)$ we define the zero set of $u$, denoted $Z(u)$, by $Z(u)=\{g \in G: u(g)=0\}$. Note that since $u$ is continuous, $Z(u)$ is closed. Also for a closed ideal $J \subset A(G)$ we define the hull of $J$, denoted $h(J)$, as follows:

$$
h(J)=\{x \in G: f(x)=0 \forall f \in J\} .
$$


Given a closed set $E \in G$ we define the closed ideal $\mathcal{I}(E)$ by

$$
\mathcal{I}(E)=\{f \in A(G): f(x)=0 \forall x \in E\} .
$$

Let $G_{d}$ denote the group $G$ with the discrete topology. The coset ring of $G$, denoted $\Omega(G)$, is the smallest ring of sets, closed under finite unions, intersections and translation containing all subgroups, and we define the closed coset ring of $G$, denoted $\Omega_{c}(G)$, to consist of all elements of $\Omega(G)$ which are closed in $G$. Applying the results of the last section, we have:

Theorem 5. Let $G$ be an amenable group. If $\mathcal{J}$ is a closed ideal such that $\mathcal{J}^{\perp}$ is completely complemented in $A(G)^{*}$, then $h(\mathcal{J}) \in \Omega_{c}(G)$. In particular if $\mathcal{J}$ is completely complemented, then $h(\mathcal{J}) \in \Omega_{c}(G)$.

Proof. Since $G$ is amenable, $A(G)$ is operator amenable ([20]). Therefore by Theorem $4, \mathcal{J}^{\perp}$ complemented by a completely bounded projection implies that $\mathcal{J}$ has a bounded approximate identity $\left\{u_{\alpha}\right\}$. It follows from [9] that $u_{\alpha} \in B\left(G_{d}\right)$ and $\left\|u_{\alpha}\right\|_{B\left(G_{d}\right)}=\left\|u_{\alpha}\right\|_{A(G)}$. Let $u$ be a weak-* limit point of this bounded approximate identity in $B\left(G_{d}\right)$. It is routine to show that $u$ is an idempotent in $B\left(G_{d}\right)$ with $Z(u)=h(\mathcal{J})$. By Host's Idempotent Theorem [13] we can conclude that $Z(u) \in \Omega(G)$ and hence $h(J) \in \Omega_{c}(G)$ (see also [10]).

Remark. We note that Rosenthal's result ([18]) is for bounded projections, as opposed to completely bounded projections. However if $G$ is Abelian, then $A(G) \cong$ $L^{1}(\hat{G})$ which is known to have the $M A X$ operator space structure. In this case it follows that every bounded projection is automatically completely bounded, and hence in particular we see that Theorem 5 is a true generalization of Rosenthal's result to the non-Abelian case. Unfortunately, for the Fourier algebra to have the $M A X$ operator space structure its dual space $V N(G)$ will have the $M I N$ operator space structure (see [3]), from which it follows that $V N(G)$ is a commutative operator algebra and hence $G$ is Abelian (see [4]). Thus we cannot conclude that every bounded projection is automatically completely bounded for arbitrary $G$.

Also note that in general the converse of Theorem 5 is false, even in the Abelian case. (See [1] for the case $G=\mathbf{R}$.) However, in the discrete case the converse does hold and we have the following characterization of ideals complemented by completely bounded maps.

Corollary 6. Let $G$ be an amenable discrete group. Then $\mathcal{J}$ is completely complemented if and only if $h(\mathcal{J}) \in \Omega_{c}(G)$.

Proof. $(\Rightarrow)$ This follows immediately from Theorem 5 .

$(\Leftarrow)$ If $h(\mathcal{J})=E \subset \Omega_{c}(G)$, then the characteristic function of $E$, denoted $1_{E}$, is an element of $B(G)([9])$. Thus the map $P(u)=u \cdot 1_{E}$ from $A(G)$ onto $\mathcal{J}$ is a completely bounded projection of $A(G)$ onto $\mathcal{I}(h(\mathcal{J}))$. Since this is a set of spectral synthesis it follows that $\mathcal{I}(h(\mathcal{J}))=\mathcal{J}$.

The following example due to Leinert shows that the condition on the amenability of the group is necessary for the previous corollary. Let $F_{2}$ be the free group on $\{a, b\}$. It is well known that $F_{2}$ is not an amenable group. Let $E=\left\{a^{n} b^{n}: n=\right.$ $1,2, \ldots\}$. Then the characteristic function $1_{E}$ of $E$ is completely bounded (see [15] for details); however $E$ is clearly not an element of the coset ring. In particular, $\mathcal{I}(E)$ provides an example of a completely complemented ideal in $A\left(F_{2}\right)$ whose hull is not in the coset ring. 


\section{REFERENCES}

[1] Alspach, D.E. and Matheson, A., Projections onto translation invariant subspaces of $L^{1}(R)$, Trans. Amer. Math. Soc. 277 (1984), 815-823 MR 85e:46017

[2] Blecher, D.P., A completely bounded characterization of operator algebras, Math. Ann. 303, (1995) 227-239 MR 96k:46098

[3] -, The standard dual of an operator space, Pacific J. Math. 153 (1992), 15-30 MR 93d: 47083

[4] —, Commutativity in operator algebras, Proc. Amer. Math. Soc. 109 (1990), 709-715 MR 90k: 46128

[5] Blecher, D.P., and Paulsen V.I., Tensor products of operator spaces, J. Funct. Anal. 99 (1991) 262-292 MR 93d:46095

[6] Curtis, P.C. Jr. and Loy R.J., Amenable Banach algebras, J. London Math. Soc. 40 (1989) 89-104 MR 90k:46114

[7] Effros, E.G. and Ruan, Z-J, On matricially normed spaces, Pacific J. Math 132 (1988), 243-264 MR 90a:46150

[8] Effros, E.G and Ruan, Z-J, On the abstract characterization of operator spaces, Proc. Amer. Math. Soc. 119 (1990), 579-584 MR 94g:46019

[9] Eymard P., L'algebra de Fourier d'un groupe localement compact, Bull. Soc. Math. de France 92 (1964), 181-236. MR 37:4208

[10] Forrest, B.E., Amenability and bounded approximate identities in ideals of $A(G)$, Illinois J. Math 34 (1990) 1-25 MR 92e:43003

[11] Johnson, B.E. Cohmology in Banach Algebras, Memoirs Amer. Math. Soc. 127 (1972) MR 51:11130

[12] —, Non amenability of the Fourier algebra of a compact group, J. London Math. Soc. (2) 50 (1994) 361-374 MR 95i:43001

[13] Host, B., Le theorem des idempotents dans B(G), Bull. Math. France 114 (1986), 215-223 MR 88b:43003

[14] Khelemskii, A. Ya., Flat Banach modules and amenable algebras, Trans. Moscow Math. Soc. (1984); Amer. Math. Soc. Translations (1985) 199-224 MR 86g:46108

[15] Leinert, M. Faltungsoperatorem auf gewissen diskreten gruppen, Studia. Mat. 62 (1973) 149-158 MR 50:7954

[16] Lui, T-S, van Rooij A. and Wang, J.K., Projections and approximate identities for ideals in group algebras, Trans. Amer. Math. Soc. 175 (1973) 469-482

[17] Paulsen, V.I., Representations of function algebras, abstract operator spaces and Banach space geometry, J. Funct. Anal. 109 (1992) 113-129 MR 93h:46001

[18] Rosenthal, H.P., Projections onto translation invariant subspaces of $L^{p}(G)$, Mem. Amer. Math. Soc. No. 63 (1966) MR 35:2080

[19] Rudin, W., Projections on invariant subspaces, Proc. Amer. Math. Soc. 13 (1962) 429-432 MR 25:1460

[20] Ruan, Z-J., The operator amenability of $A(G)$, Amer J. Math. 117 (1995) 1449-1476 MR 96m: 43001

[21] Ruan, Z-J., and Xu, G., Splitting properties of operator bimodules and operator amenability of Kac algebras (1996) preprint

Department of Pure Mathematics, University of Waterloo, Waterloo, Ontario, CANADA N2L 3G1

E-mail address: pwood@barrow.uwaterloo.ca 